\title{
Loss of E-cadherin in the vicinity of necrosis in colorectal carcinomas: Association with NFkB expression
}

\author{
NEKTARIA SIMIANTONAKI ${ }^{1,3^{*}}$, URSULA KURZIK-DUMKE ${ }^{2 *}$, GEORGIA KARYOFYLLI $^{1}$, \\ CAREN JAYASINGHE ${ }^{1,4}$ and CHARLES JAMES KIRKPATRICK ${ }^{1}$ \\ ${ }^{1}$ Institute of Pathology, Johannes Gutenberg University, Langenbeckstrasse 1; ${ }^{2}$ Institute of Medical Microbiology \\ and Hygiene, Comparative Tumor Biology Group, Johannes Gutenberg University, \\ Obere Zahlbacher Strasse 67, D-55131 Mainz, Germany
}

Received March 20, 2007; Accepted May 2, 2007

\begin{abstract}
The transcription factor $\mathrm{NF}_{\mathrm{B}} \mathrm{B}$ regulates the expression of several tumor-related molecules associated with tumor progression and metastasis. However, the precise mechanisms by which its activation mediates these processes in diverse tumors are unknown. In this study we determined the expression of $\mathrm{NF \kappa B}$ in various colorectal carcinoma cell lines, in a series of 90 non-metastatic and metastatic colorectal tumors and in an in vitro 3D-spheroid model of HT-29 cells simulating morphological hallmark of these adenocarcinomas, namely neoplastic glandular nests around a necrotic center. We show that the inactive cytoplasmic $\mathrm{NF} \kappa \mathrm{B}$ form is evidently up-regulated in the tumor epithelium, especially in the metastatic cases, as compared to normal tissue. We found that in situ nuclear NFKB staining is characteristic for cells that are still viable but dissociated from the surrounding cohesive tumor tissue and destined to die. Evidence for a possible association between $\mathrm{NF \kappa B}$ expression and loss of cell adhesion mediated by E-cadherin function has been provided in vivo and in vitro using the HT-29 3Dspheroid model. In both cases, we found a strong correlation between activation of $\mathrm{NF \kappa B}$ and loss of E-cadherin expression. Considering the fact that cancer cell necrosis plays a crucial
\end{abstract}

Correspondence to: Dr Nektaria Simiantonaki ${ }^{3}$ Present address: Institute of Pathology Klinikum Leverkusen, Am Gesundheitspark 11, D-51137 Leverkusen, Germany

E-mail: simiantonaki@klinikum-lev.de

Professor Ursula Kurzik-Dumke, Institute of Medical Microbiology and Hygiene, Comparative Tumor Biology Group, Obere Zahlbacher Strasse 67, D-55131 Mainz, Germany

E-mail:kurzik@mail.uni-mainz.de

Present address: ${ }^{4}$ Institute of Pathology Klinikum Leverkusen, Am Gesundheitspark 11, D-51137 Leverkusen, Germany

${ }^{*}$ Contributed equally

Key words: NFкB, E-cadherin, necrosis, colorectal cancer role in metastasis, $\mathrm{NF \kappa B}$ activation mediated by loss of Ecadherin may represent an essential, even initial event in this process. Furthermore, we present in vitro data implicating LPS, the endotoxin of gram-negative bacteria, in the triggering of NFKB up-regulation. Thus, release of bacterial endotoxin may essentially contribute to the progression of colon cancer in vivo.

\section{Introduction}

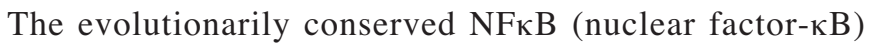
protein was originally discovered in $\mathrm{B}$ cells as a nuclear factor binding to the enhancer of the kappa light chain of immunoglobulin (1). Currently its expression in all cell types of diverse species from the fruit fly Drosophila to man has

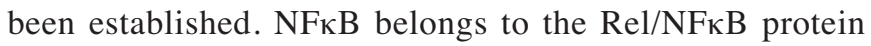
family encompassing NFKB1 (p50), NFKB2 (p52) and the Rel proteins RelA (p65), RelB and c-Rel (2-4). The classical $\mathrm{NF} \mathrm{B}$ occurs as a dimer consisting of two proteins, p50 and p65. In normal cells, except for proliferating $\mathrm{T}$ and $\mathrm{B}$ cells and thymocytes, monocytes and astrocytes, it forms an inactive complex with inhibitory proteins, members of the $\mathrm{I} \kappa \mathrm{B}$ (inhibitor of nuclear factor- $\kappa \mathrm{B}$ ) family. Already these features indicate association of $\mathrm{NF}_{\kappa} \mathrm{B}$ with proliferative processes. In agreement with that it has been found that in most tumor cell lines, derived from both hematopoietic and solid tumors, NFKB is active and that its inappropriate activation mediates both tumorigenesis and inflammation.

The inactive $\mathrm{I} \kappa \mathrm{B}$-bond $\mathrm{NF} \kappa \mathrm{B}$ form is retained in the cytoplasm. To translocate into the nucleus $\mathrm{NF \kappa B} / \mathrm{I} \kappa \mathrm{B}$ binding is released by rapid $\mathrm{I}_{\kappa} \mathrm{B}$ phosphorylation and degradation via the ubiquitin-proteosome pathway. As is currently known the active $\mathrm{NF \kappa B}_{\mathrm{B}}$ induces the expression of a variety of genes regulating the immune response, growth, cell survival and apoptosis by binding to their specific $\kappa \mathrm{B}$-elements. Thus, proper development of cells depends on precise activation/ inactivation of this transcriptional regulator.

As mentioned above, a constitutive activation of $\mathrm{NF \kappa B}$ has been reported in nearly all tumor tissues including carcinomas of the gastrointestinal tract. Since the expression of oncogenes such as Ras and c-myc is mediated by NFkB, its constitutive activation has numerous consequences for tumor development 
and progression. Furthermore, angiogenesis, tumor invasion and metastasis are regulated by numerous NFKB-regulated gene products such as matrix metalloproteinases, chemokines, growth factors and the adhesion molecules ICAM-1, VCAM-1 and E-selectin. In human prostate cancer cells inactivation of $\mathrm{NF \kappa B}$ results in suppression of angiogenesis, invasion and metastasis (5) and in a murine lung alveolar carcinoma cell line, Line 1, in down-regulation of prometastatic factors and up-regulation of antimetastatic factors preventing intravasation of tumor cells (6). These data provide a strong indication that NFkB essentially contributes to cancer.

The past few years have brought tremendous progress in understanding the mechanisms of NFKB-modulated responses and their significance in tumor behaviour. However, the precise mechanisms by which $\mathrm{NF \kappa B}$ activation mediates progression and metastasis of specific cancers still remain to be elucidated. In this study we examined the relevance of $\mathrm{NF} \kappa \mathrm{B}$ for colorectal cancer. In a series of 90 non-metastatic, lymphogenous-metastatic and haematogenous-metastatic colorectal carcinomas, we determined the expression pattern of NFKB in the context of the metastatic status of these tumors. Since we found significant up-regulation of cytosolic $\mathrm{NF} \kappa \mathrm{B}$ in tumor epithelium and its nuclear accumulation in dissociated tumor cells we examined a possible correlation between the expression levels of nuclear NFKB and the adhesion molecule E-cadherin in colorectal carcinoma specimens. We examined the reproducibility of the data obtained by in situ analysis in a 3D in vitro culture model using tumor spheroids, which more closely imitate the in vivo situation than conventional monolayer culture systems. Furthermore, in accordance with our previous data, suggesting that the bacterial lipopolysaccharide (LPS) causes up-regulation of adhesion molecules, which play an essential role in metastasis, we examined the influence of LPS stimulation on the expression of $\mathrm{NF \kappa B}$ in various colorectal carcinoma cell lines (7).

\section{Materials and methods}

Cultivation of adherent tumor cells and LPS stimulation. The human colorectal cell lines SW837, HRT18, CX-1, CX-2, SW620, SW948, HT-29 and CaCo2 were grown in RPMI-1640 medium supplemented with Glutamax (Sigma), 10\% heat deactivated FCS (Gibco), 1\% penicillin (Gibco) and 1\% streptomycin (Gibco) at $37^{\circ} \mathrm{C}$ in a humidified incubator containing $5 \% \mathrm{CO}_{2}$. LPS stimulation was performed by replacing the growth medium of subconfluent cultures with medium supplemented with LPS derived from E. coli (Sigma), $1 \mu \mathrm{g} / \mathrm{ml}$. After $4 \mathrm{~h}$ the cells were harvested and used for preparation of extracts for expression analysis.

$3 D$ culture of HT-29 cells as spheroids. The HT-29 cells were harvested in the exponential growth phase ( $80 \%$ confluency). After washing once in medium the cells were seeded on agarose-coated (1\%) 96-multiwell plates at a concetration of 1000 cells $/ 200 \mu \mathrm{l}$. Within 5 days of incubation at $37^{\circ} \mathrm{C}$ the cells formed compact aggregates. For expression studies only cells in the plateau phase of growth were used. This state is reached after cultivation of the spheroids for two weeks. After two weeks the spheroids were harvested, formalin- fixed, embedded in paraffin and processed for immunohistochemistry.

Tissue samples. The colorectal tissue samples used in this study were obtained from 90 patients undergoing elective surgery for colorectal cancer at the University of Mainz during the years 1995-1999. The investigation of these tissues was in accordance with the rules of the responsible state ethics committee. The morphological classification of the carcinomas was conducted according to WHO specifications. The tumors were staged following the guidelines of the TNM Classification of Malignant Tumors. With respect to the T status all tumors investigated were T3 and moderately differentiated $(\mathrm{G} 2)$ and were separated into three groups according to metastatic status. The first group included 30 cases without tumor metastasis to regional lymph nodes or distant organs (N0/M0). Among the remaining 60 metastatic cases 30 were characterized by lymphogenous $(\mathrm{N}+)$ and 30 by haematogenous metastases $(\mathrm{M}+)$. For all samples investigated follow-up data were obtained from hospital charts and by corresponding with the physicians in charge during a period of 5 years after surgery.

Antibodies. Primary antibodies: mouse monoclonal NF-кB (RelA) (F-6, Santa Cruz Biotechnology, Inc.), mouse monoclonal anti-E-cadherin (BioGenex) and rabbit polyclonal anti-ß-actin (Serva).

Western blot analysis. Protein extracts from harvested human cells and normal and tumor epithelia derived from patients undergoing surgical resection of colorectal carcinomas were prepared in TKM buffer (50 mM Tris, $\mathrm{pH} 7.5 / 150 \mathrm{mM} \mathrm{KCl} /$ $5 \mathrm{mM} \mathrm{MgCl}_{2}$ ) using a Dounce homogenizer. The total protein content was determined using the Bio-Rad Protein Assay (Bio-Rad Laboratories $\mathrm{GmbH}$ ). For Western blotting aliquots from each sample containing $20 \mu \mathrm{g}$ of total protein were separated on sodium dodecyl sulphate-polyacrylamide gels SDS-PA $(10 \%)$ and then transferred to polyvinylfluoride (PVDF) membranes (Immobilen-P, Millipore Corp.) in accordance with standard procedures. Incubation with the primary antibodies to NFKB (RelA) and B-actin was performed overnight by $4^{\circ} \mathrm{C}$. Immunodetection was performed using the alkaline phosphatase (AP) conjugated anti-mouse and antirabbit IgG (Sigma). As AP substrate a mix containing $0.45 \%$ nitroblue tetrazolium (NBT) (Serva) and $0.35 \%$ bromo-4chloro-3-indolyl phosphate toluidinium salt (X-Phosphat) (Serva) in AP buffer (100 mM NaCl/50 mM MgCl $2 / 100 \mathrm{mM}$ Tris, $\mathrm{pH}$ 9.2) was used. Primary antibodies were used in a 1:100 dilution. Secondary antibodies were used at concentrations recommended by the suppliers.

Immunohistochemistry/cytochemistry. All immunohistochemical reactions were conducted using formalin-fixed and paraffin-embedded samples. After deparaffination the samples were treated in a microwave oven in EDTA buffer for $15 \mathrm{~min}$. Incubation with the primary antibodies to NFKB (RelA) and E-cadherin, and the secondary antibodies, horse anti-mouse biotinylated IgG (Vector Laboratories, Inc.) were carried out using the Vectastain Elite reagent (Vector Laboratories, Inc.). Anti-NFKB was used at a dilution of 
(A)

\begin{tabular}{|c|c|c|c|c|c|}
\hline $\mathrm{N}$ & $\mathrm{T}$ & $\mathrm{N}$ & $\mathrm{T}$ & $\mathrm{N}$ & $\mathrm{T}$ \\
\hline$=$ & - & & 8 & & 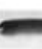 \\
\hline r & 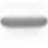 & $=$ & - & - & - \\
\hline
\end{tabular}

(B)

\begin{tabular}{|c|c|c|c|c|c|c|c|c|c|}
\hline \multicolumn{2}{|c|}{1} & & 2 & \multicolumn{2}{|c|}{3} & \multicolumn{2}{|c|}{4} & \multicolumn{2}{|c|}{5} \\
\hline $\mathrm{N}$ & $\mathrm{T}$ & $\mathrm{N}$ & $\mathrm{T}$ & $\mathrm{N}$ & $\mathrm{T}$ & $\mathrm{N}$ & $\mathrm{T}$ & $\mathrm{N}$ & $\mathrm{T}$ \\
\hline e & & - & 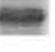 & & 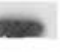 & - & $\rightarrow$ & $=$ & $\Rightarrow$ \\
\hline$\Leftrightarrow$ & & & 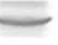 & - & - & & $=$ & & \\
\hline
\end{tabular}

Figure 1. Detection of NFKB (RelA) in human non-metastatic (A) and metastatic (B) primary colorectal carcinomas by Western blotting. Aliquots of tissue homogenates containing $20 \mu \mathrm{g}$ of total protein were loaded onto the SDS-PA gel $(10 \%)$ and after separation transferred to PVDF membrane. Staining with anti- $\beta$-actin was performed as a control for loading (bottom blots respectively). ( $\mathrm{N}$, normal epithelium; $\mathrm{T}$, tumor epithelium).

1:1500, anti-E-cadherin was used at a dilution 1:20. All secondary antibodies employed in this study were used at a dilution 1:200. Sections were counterstained with Mayer's hematoxylin. To prove the specificity of the immunoreactions every colorectal carcinoma sample $(n=90)$ was stained solely with the secondary antibody. Control reactions were performed for each of the primary antibodies used. Immunostaining reactions of each sample were evaluated by three authors independently (N. Simiantonaki, C. Jayasinghe, G. Karyofylli). The evaluation of the immunohistochemical staining was performed in accordance with a previously established scoring method, classifying the tumors with respect to the cytoplasmic staining intensity into three groups (weak, moderate and strong staining). In those cases where heterogeneous staining was observed within the same sample that level of staining which was visible in more than $50 \%$ of the cells was chosen for the classification into a defined group.

Statistical analyses. The evaluation of data concerning association of staining intensity with tumor stage was assessed using $\chi^{2}$ (Fisher's exact test). $\mathrm{P}<0.05$ was considered to be significant in all statistical analyses.

\section{Results}

The level of cytoplasmic $N F \kappa B$ is elevated in colon cancer epithelia. To elucidate the relevance of $\mathrm{NF \kappa B}$ for colorectal cancer progression we determined its expression in nonmetastatic (N0/M0, 3 cases) and metastatic ( $\mathrm{N}+$ or/and $\mathrm{M}+$, 5 cases) colon carcinomas by Western blotting (Fig. 1) and by immunohistochemistry (Fig. 2). All specimens examined were derived exclusively from the surface of the tumor. Furthermore, all samples chosen for this analysis were comparable regarding the amount of the tumor cells and the adjacent tissue including the surrounding cells positive for NFkB. As shown in Fig. 1 all tumor cases are characterized by a significant increase of NFKB expression as compared to normal tissue. The up-regulation is independent of the
Table I. Expression of NFKB (RelA) in non-metastatic (N0/M0), lymphogenous-metastatic $(\mathrm{N}+)$ and haematogenous-metastatic $(\mathrm{M}+)$ colorectal carcinomas. ${ }^{\mathrm{a}}$

\begin{tabular}{lcccr}
\hline $\begin{array}{l}\text { Colorectal } \\
\text { carcinomas }\end{array}$ & $\mathrm{n}$ & $\begin{array}{c}\text { Weak } \\
(\%)\end{array}$ & $\begin{array}{c}\text { Moderate } \\
(\%)\end{array}$ & \multicolumn{1}{c}{$\begin{array}{c}\text { Strong } \\
(\%)\end{array}$} \\
\hline N0/M0 & 30 & $7(23)$ & $17(57)$ & $6(20)$ \\
N+ & 30 & $8(27)$ & $9(30)$ & $13(43)$ \\
M+ & 30 & $7(23)$ & $9(30)$ & $14(47)$ \\
\hline
\end{tabular}

${ }^{a}$ Considering the expression levels, the cases examined were separated into three groups. Group 1 is characterized by a weak NFKB (RelA) expression (cf. Fig. 2A). Group 2 shows moderate NFkB (RelA) level (cf. Fig. 2B) whereas group 3 is characterized by a strong stain (cf. Fig. 2C). n, number of cases examined.

metastatic status of the tumors. The non-neoplastic tissue of 6 of the cases investigated showed a weak to moderate $\mathrm{NF} \kappa \mathrm{B}$ expression whereas in 2 cases the staining for $\mathrm{NF \kappa B}$ was negative.

To verify the data gained by Western blotting a large pool of colorectal carcinomas, 90 in all, was investigated by immunohistochemistry (Table I). The cases examined were grouped according to their metastatic status into three groups. The first group included 30 non-metastatic cases (N0/M0). The remaining 60 cases had metastases, 30 of these samples being characterized by lymphogenous $(\mathrm{N}+)$ and 30 by haematogenous $(\mathrm{M}+)$ metastases. With respect to the $\mathrm{T}$ status all tumors investigated were defined as T3 and moderately differentiated (G2). This selection was performed with regard to a potential relationship between the expression level of $\mathrm{NFKB}_{\mathrm{B}}$ and the metastatic status of the tumors. As shown in Table I all cases investigated were positive for cytoplasmic NFKB. Considering the expression intensity weak, moderate, strong - the cases were classified into three groups (Table I, Fig. 2A-C). The data presented in Table I indicate that cancer progression in the colon correlates with increase of cytosolic NFKB expression. Whereas only $20 \%$ of the N0/M0 tumors showed high cytosolic NFKB levels, $43 \%(\mathrm{p}=0.09)$ of the lymphogenous- and $47 \%(\mathrm{p}=0.05)$ of the haematogenous-metastatic cases gave strong expression. In contrast, the non-neoplastic tissue revealed only a weak cytoplasmic immunostaining (Fig. 2D).

In colon cancer nuclear expression of $N F \kappa B$ accompanies loss of E-cadherin. As shown above, the increased cytosolic $\mathrm{NF} \mathrm{B}$ expression was characteristic for all 90 tumor samples examined, independent of their metastatic status. Seventy-eight samples $(88 \%)$ showed additionally nuclear expression. Notably, in each of these specimens only few (about 5-10\%) cells showed endonuclear NFkB localization.

Abundant central necrosis in the atypical glands is a characteristic feature of colonic adenocarcinomas (Fig. 3A1). In our study 65 (72\%) of the 90 tumors examined were characterized by this histopathological morphology. Forty-nine $(75 \%)$ of these samples showed additionally to the cytoplasmic 

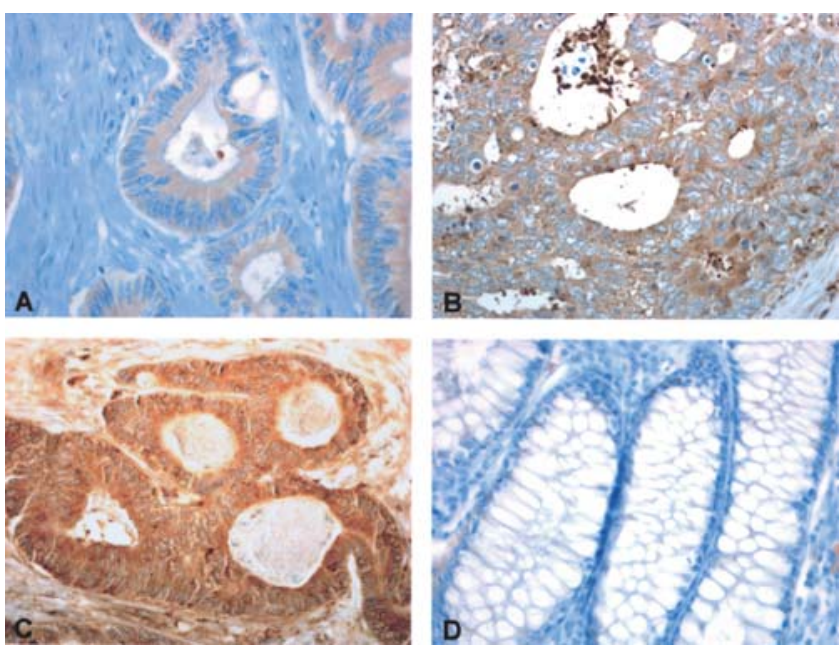

Figure 2. The level of cytosolic NFkB (RelA) is up-regulated in colorectal carcinomas. A, Specimen characterized by a weak stain. B, Example of a moderate stain. C, Specimen showing a strong immunoreactivity to $\mathrm{NFkB}$ (RelA). D, NFKB (RelA) expression in non-neoplastic colon mucosa. The sections were counterstained with Mayer's hematoxylin. Magnification x400.

staining nuclear NFkB localization in cells, which were still vital but dissociated from the surrounding cohesive tumor tissue (Fig. 3A2) undergoing cell death. This morphological finding implies a correlation between the activation of $\mathrm{NF \kappa B}$, loss of adhesion and the necrotic process. Since tissue integrity is principally mediated by the cell-cell adhesion molecule E-cadherin, we determined its expression in the aforementioned 65 cases. Interestingly, $43(66 \%, p=0.0008)$ of these samples
Table II. Relationship between nuclear expression of $\mathrm{NF \kappa B}$ (RelA) and E-cadherin in the dissociated cells surrounding central necroses established by evaluation of 65 colorectal carcinomas $(\mathrm{p}=0.0008)$.

\begin{tabular}{lrcc}
\hline & NFKB $^{+}$ & NFkB $^{-}$ & Total \\
\hline E-cadherin positive & 6 & 9 & 15 \\
E-cadherin negative & 43 & 7 & 50 \\
Total & 49 & 16 & 65 \\
\hline
\end{tabular}

showed loss of E-cadherin (Table II and Fig. 3A3) (8). In a few samples loss of E-cadherin was observed within tumor complexes which were not associated with the central necrosis (Fig. 3B1-3). Here mainly an increase of cytosolic NFKB expression and nuclear staining of only a few tumor cells was found. Additionally, in 10 adenocarcinomas with local mucus production a positive nuclear $\mathrm{NF} \kappa \mathrm{B}$ expression was found in the E-cadherin-negative tumor cells present in the mucus (Fig. 3C1-3). The association of NFkB activation and loss of E-cadherin was independent of the metastatic status of the examined cases.

Nuclear expression of $N F \kappa B$ is associated with loss of Ecadherin in the HT-29 spheroid model. Adenocarcinomas of the colon are characterized by a typical morphological feature, namely central necrosis surrounded by neoplastic glandular nests. As described previously 3D spheroid cultures of tumor
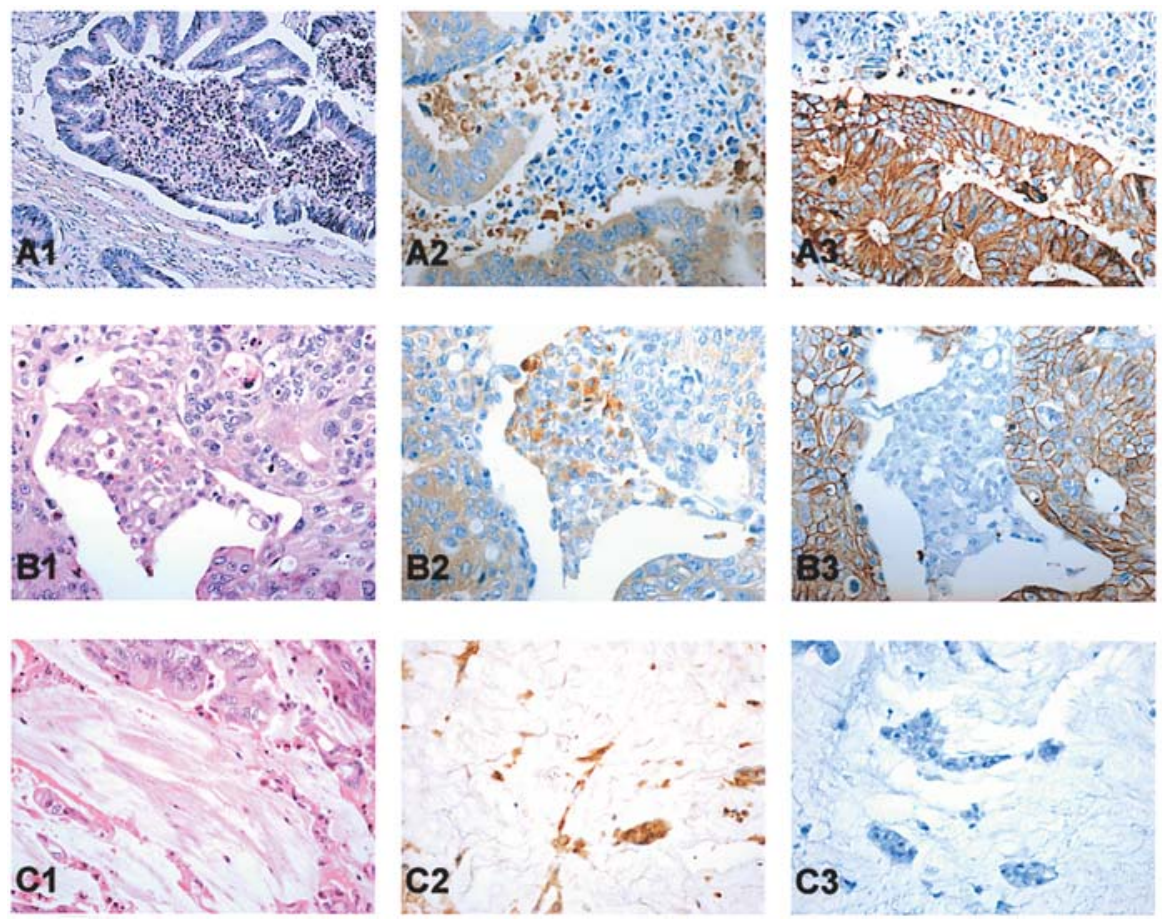

Figure 3. In colorectal carcinomas nuclear location of NFKB (RelA) expression correlates with loss of E-cadherin. A1, Morphology of a colon adenocarcinoma characterized by an area of central necrosis surrounded by neoplastic glandular nests stained with hematoxylin. A2, Nuclear NFkB (RelA) staining in still viable cells but dissociated from the surrounding cohesive tumor. A3, E-cadherin is expressed in the cohesive tumor but not in the dissociated cells positive for NFKB (RelA). B1, Compact tumor complex stained with hematoxylin. B2, Strong NFkB (RelA) staining is visible in the cytoplasm. A few cells show also nuclear staining. B3, Cells expressing NFKB (RelA) are negative for E-cadherin. C1, Adenocarcinoma with local mucus production stained with hematoxylin. C2, Cells floating in mucus are positive for nuclear NFKB (RelA). C3, Cells expressing NFKB (RelA) are negative for E-cadherin. Magnification x160 (A1) and x400 (A2-C3). 

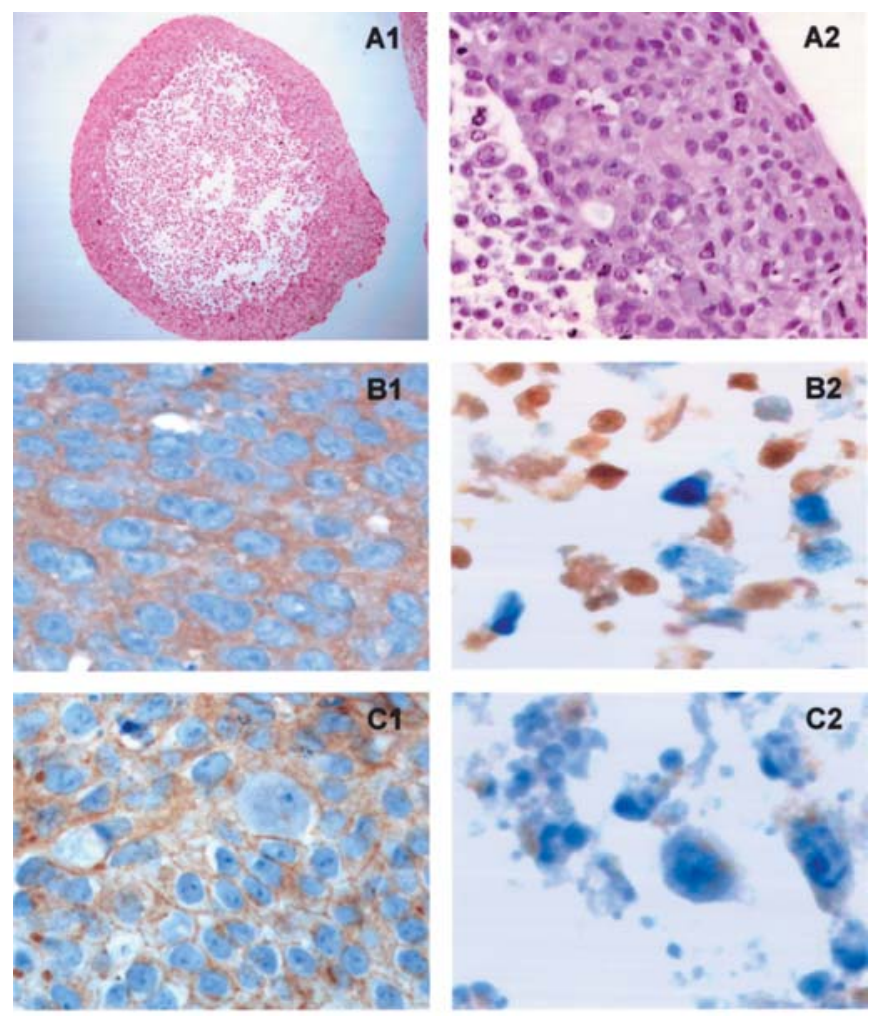

C2 shown in Fig. $4 \mathrm{~A}$ in the inner part of HT-29 spheroids a necrotic centre is formed during the plateau period of growth. Around the necrosis a non-proliferative area with dissociated but still viable cells is present, whereas the outer region consists of tightly packed cells. This characteristic morphology mimics precisely the in situ situation. Similar to that shown in situ, HT-29 spheroids express NFкB in the cytoplasm of all viable cells and in the nucleus of some dissociated cells outside the compact cell area (Fig. 4B). Interestingly enough, these dissociated cells also show loss of E-cadherin, whereas in the compact outer region of the spheroid a positive,

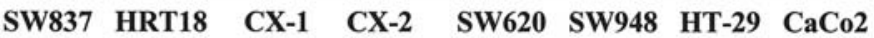

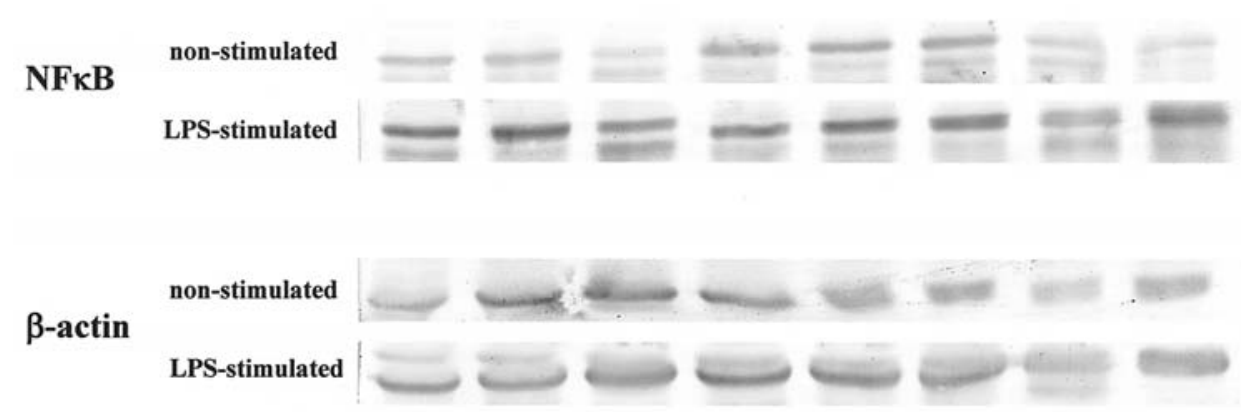

Figure 5. Detection of NF-kB (RelA) in non-stimulated and LPS-stimulated human colorectal carcinoma cell lines by Western blotting. Aliquots of cell homogenates containing $20 \mu \mathrm{g}$ of total protein were loaded onto the SDS-PA gel (10\%) and after separation transferred to PVDF membrane. Staining with anti-B-actin was performed as a control for loading.
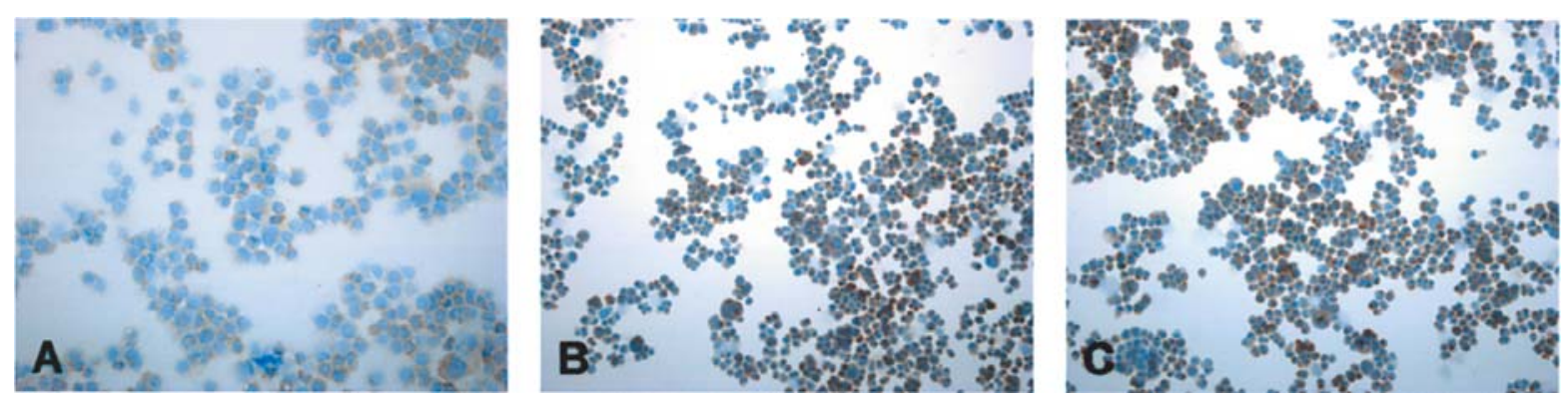

Figure 6. LPS-stimulation of $\mathrm{CaCo} 2$ cells results in up-regulation of the cytosolic NFkB (RelA). A, Weak NFKB (RelA) cytoplasmic staining in native, unstimulated $\mathrm{CaCo} 2$ cells. B, Strong cytosolic immunoreactivity to NFKB (RelA) $1 \mathrm{~h}$ after LPS-stimulation. C, The strong cytosolic immunoreactivity to NFkB (RelA) persists $4 \mathrm{~h}$ after LPS stimulation. The cells were counterstained with Mayer's hematoxylin. Magnification x160. 
membranous staining of this adhesion molecule is visible (Fig. 4C).

LPS induces $N F \kappa B$-expression in colorectal carcinoma cells. Since exposure of certain cell types to LPS leads to activation of NFKB, we asked whether this is true also for cell lines derived from colorectal carcinomas (2). We examined by Western blot the expression of $\mathrm{NF \kappa B}$ in non-stimulated and LPS-stimulated SW837, HRT18, CX-1, CX-2, SW620, SW948, HT-29 and CaCo2 cells. As shown in Fig. 5 all eight cell lines are positive for $\mathrm{NF \kappa B}$. Its expression level differs from very weak in the well differentiated $\mathrm{CaCo} 2$ cells to moderate or high in the less differentiated cell lines. Interestingly, LPS treatment of the $\mathrm{CaCo} 2$ cells, characterized by lower constitutive NFKB levels, caused a marked increase of its expression (Fig. 5). In the cell lines characterized by relatively high amounts of constitutive $\mathrm{NFKB}$ a significant response to LPS was also observed. To elucidate whether LPS stimulation influences not only the expression level but also the subcellular localization of NFKB we examined its distribution in $\mathrm{CaCo} 2$ cells by immunocytochemistry. Considering the kinetics of the translocation process we monitored the expression pattern at five different time-points $(0,30 \mathrm{~min}, 1,2$ and $4 \mathrm{~h}$ after LPS exposure). As shown in Fig. 6 in both the non-stimulated (A) and LPS-stimulated $\mathrm{CaCo} 2$ cells ( $\mathrm{B}$ and $\mathrm{C}$ ) only a cytoplasmatic staining is apparent. A significant increase of NFKB level was visible already one hour after stimulation.

\section{Discussion}

The pivotal role of the transcription factor $\mathrm{NF \kappa B}$ in cell proliferation and survival has been documented by numerous studies. Since both processes are strongly associated with neoplastic transformation and progression of malignancies, the finding that $\mathrm{NF \kappa B}$ expression is altered in diverse neoplastic cells and tissues derived from both hematopoietic and solid tumors was not surprising. Currently it is known that both $\mathrm{NF \kappa B}$ and its regulators are associated with tumor-related processes, such as neoplastic transformation, proliferation, apoptosis, angiogenesis and metastasis. However, the mechanisms leading to its aberrant activation/silencing and cellular trafficking are not understood in the special context of defined cancers. Furthermore, the triggers of these processes have not yet been identified.

In this study it is demonstrated that colorectal adenocarcinomas are generally characterized by elevated levels of the inactive cytosolic NFKB. Nevertheless, this phenomenon was found to be strongly linked to the metastatic status of the tumors investigated, as both the lymphogenous- and haematogenous-metastatic tumors were affected. Thus, we suggest that up-regulation of $\mathrm{NF \kappa B}$ is associated with the aggressiveness of the tumors. Nuclear localization of the transcription factor was detected exclusively in cells dissociated from the central necrosis of the adenocarcinomas. Our results confirm the predominant cytosolic and exclusively focal nuclear localization of $\mathrm{NF \kappa B}$ found in colorectal, pancreatic, gastric and prostate carcinomas by other investigators (10-15). In agreement with our suggestion of a correlation between the up-regulation of $\mathrm{NF \kappa B}$ and the aggressiveness of the tumors, Evertsson and Sun found NFkB activation in mucinous colorectal adenocarcinomas with a poor prognosis and Kojima et al in the more progressed T3+T4 cases $(10,16)$. Additional support is provided by correlation of $\mathrm{NF \kappa B}$ and VEGF expression, suggesting a link between $\mathrm{NF \kappa B}$ and tumor angiogenesis (12). NFKB inhibitory action in apoptosis has also been found in colorectal carcinomas $(11,17)$. With regard to the nuclear staining of the dissociated cells in the vicinity of the central necrosis the question arises whether the translocation of NFKB into the nucleus results directly from loss of homeostasis between the active and the non-active form of the transcription factor. Furthermore, whether this loss of balance is due to the aberrant up-regulation of NFkB expression and which molecular/genetic events are responsible for it, remain unclear.

Previously, we identified LPS, the endotoxin of intestinal Gram-negative bacteria, as a pivotal stimulus increasing the metastatic potential of colorectal cancer $(7,18)$. Therefore, in the context of the present studies the expression of NFKB was examined in colorectal tumor cell lines SW837, HRT18, CX-1, CX-2, SW620, SW948, HT-29 and CaCo2 after LPS treatment. Since in all cases an increase in NFkB level has been observed, LPS may act in situ as a trigger of tumorigenic processes associated with $\mathrm{NF \kappa B}$ up-regulation. The association of LPS-mediated NFKB activation with metastasis has already been described by Luo et al (19). In an experimental murine tumor model LPS stimulation of a colon adenocarcinoma cell line caused generation of lung metastases. The metastatic growth response has been found to depend on NFkB activation in the tumor cells. The inhibition of NFKB activity converted the growth response into a death response. Andrews et al found in the human metastatic colon cancer cell line LS174T that exposure to LPS increases the adhesion of the tumor cells to the endothelium through an NFkB-dependent pathway (20).

As mentioned above, nuclear NFкB localization was observed only in cells dissociated from the cancer nests. Thus, we assumed an association between the active form of $\mathrm{NF} \mathrm{B}$ and loss of tissue integrity caused by loss of cell to cell contacts. Indeed, it has been demostrated here that the nuclear localization of $\mathrm{NF \kappa B}$ is associated with downregulation of the adhesion molecule E-cadherin. These in situ results were confirmed in vitro using a 3D-spheroid model of HT-29 cells. In keeping with our findings, a link between function(s) of NFKB and E-cadherin has been suggested by others $(21,22)$. In a mammary tumor mouse model a correlation of activation of $\mathrm{NF \kappa B}$ and loss of E-cadherin was found in cells of the more invasive phenotype (21). Activation of $\mathrm{NF \kappa B}$ caused by loss of E-cadherin was found in melanoma cell lines (22). However, our studies show that the correlation between loss of E-cadherin and up-regulation of $\mathrm{NF \kappa B}$ concerns a defined area of the tumor, namely, dissociated cells around the central necrosis of the cancer nests, suggesting that the correlation between $\mathrm{NFKB}$ activation and loss of E-cadherin may be directly associated with induction of tumor necrosis in colorectal cancer. Considering the fact that cancer cell necrosis plays a crucial role in tumor progression this observation is of great importance. It is known that necrosis of tumor cells that are located in the hypoxic core of the tumor leads to an activation of tumor- 
associated macrophages (TAM). These in turn release growth, survival and angiogenic factors that support tumor progression and increase tumor angiogenesis and invasion $(2,23)$. Furthermore the induced hypoxia potentiates these processes. However, according to the 'cross-priming' hypothesis the release of necrotic cells can reduce tolerance to tumor antigens, thereby potentiating immunosurveillance and potentially repressing tumor growth. Thus, tumor necrosis seems to have a dual and, interestingly, opposing role in the complexity of tumor events.

In conclusion, our data show that the transcription factor $\mathrm{NF} \kappa \mathrm{B}$ is of great importance for colorectal cancer. Both nonmetastatic and metastatic tumors show up-regulation of cytosolic $\mathrm{NF}_{\kappa} \mathrm{B}$ as compared to normal epithelium. Furthermore, since treatment of in vitro cultivated cells with LPS results in increase of NFKB levels, this endotoxin must be taken into consideration as a potential trigger of this process in situ. The increase of the cytoplasmic NFkB level correlates with the metastatic potential of the tumors, suggesting that the up-regulation of NFkB may be relevant to metastasis. In addition, nuclear NFKB expression strongly correlates with loss of E-cadherin expression. This correlation mainly concerns tumor cells dissociated from the necrotic core of cancer nests, thus, linking it to loss of tissue integrity and tumor necrosis, both of which are essential features in tumor progression.

\section{References}

1. Sen R and Baltimore D: Inducibility of $\kappa$ immunoglobulin enhancer-binding protein NF-kB by a posttransational mechanism. Cell 47: 921-928, 1986.

2. Aggarwal BB: Nuclear factor-kappaB: the enemy within. Cancer Cell 6: 203-208, 2004.

3. Karin M and Greten FR: NF-kappaB: linking inflammation and immunity to cancer development and progression. Nat Rev Immunol 5: 749-759, 2005.

4. Karin M: Nuclear factor-kappaB in cancer development and progression. Nature 441: 431-436, 2006.

5. Huang S, Pettaway CA, Uehara H, Bucana CD and Fidler IJ: Blockade of NF-kappaB activity in human prostate cancer cells is associated with suppression of angiogenesis, invasion, and metastasis. Oncogene 20: 4188-4197, 2001.

6. Andela VB, Schwarz EM, Puzas JE, O'Keefe RJ and Rosier RN: Tumor metastasis and the reciprocal regulation of prometastatic and antimetastatic factors by nuclear factor kappaB. Cancer Res 60: 6557-6562, 2000.

7. Simiantonaki N, Jayasinghe $\mathrm{C}$ and Kirkpatrick CJ: Effect of pro-inflammatory stimuli on tumor cell-mediated induction of endothelial cell adhesion molecules in vitro. Exp Mol Pathol 73: 46-53, 2002.
8. Hirohashi S: Inactivation of the E-cadherin-mediated cell adhesion system in human cancers. Am J Pathol 153: 333-339, 1998.

9. Santini MT and Rainaldi G: Three-dimensional spheroid model in tumor biology. Pathobiology 67: 148-157, 1999.

10. Evertsson S and Sun XF: Protein expression of NF-kappaB in human colorectal adenocarcinoma. Int J Mol Med 10: 547-550, 2002.

11. Yu LL, Yu HG, Yu JP, Luo HS, Xu XM and Li JH: Nuclear factor-kappaB p65 (RelA) transcription factor is constitutively activated in human colorectal carcinoma tissue. World $\mathrm{J}$ Gastroenterol 10: 3255-3260, 2004.

12. Yu HG, Zhong X, Yang YN, et al: Increased expression of nuclear factor-kappaB/RelA is correlated with tumor angiogenesis in human colorectal cancer. Int J Colorectal Dis 19: 18-22, 2004.

13. Wang W, Abbruzzese JL, Evans DB, Larry L, Cleary KR and Chiao PJ: The nuclear factor-kappa B RelA transcription factor is constitutively activated in human pancreatic adenocarcinoma cells. Clin Cancer Res 5: 119-127,1999.

14. Sasaki N, Morisaki T, Hashizume K, et al: Nuclear factor-kappaB p65 (RelA) transcription factor is constitutively activated in human gastric carcinoma tissue. Clin Cancer Res 7: 4136-4142, 2001.

15. Ross JS, Kallakury BV, Sheehan CE, et al: Expression of nuclear factor-kappa B and I kappa B alpha proteins in prostatic adenocarcinomas: correlation of nuclear factor-kappa B immunoreactivity with disease recurrence. Clin Cancer Res 10: 2466-2472, 2004.

16. Kojima M, Morisaki T, Sasaki N, Nakano K, Mibu R, Tanaka M and Katano M: Increased nuclear factor- $\mathrm{\kappa} B$ activation in human colorectal carcinoma and its correlation with tumor progression. Anticancer Res 24: 675-681, 2004.

17. Maihofner C, Charalambous MP, Bhambra U, Lightfoot T, Geisslinger $\mathrm{G}$ and Gooderham NJ; CRC Group: Expression of cyclooxygenase-2 parallels expression of interleukin-1beta, interleukin-6 and NF-kappaB in human colorectal cancer. Carcinogenesis 24: 665-671,2003.

18. Simiantonaki N, Kurzik-Dumke U, Karyofylli G, Jayasinghe C, Michel-Schmidt R and Kirkpatrick CJ: Reduced expression of TLR4 is associated with the metastatic status of human colorectal cancer. Int J Mol Med 20: 21-29, 2007.

19. Luo JL, Maeda S, Hsu LC, Yagita H and Karin M: Inhibition of NF-kappaB in cancer cells converts inflammation-induced tumor growth mediated by TNFalpha to TRAIL-mediated tumor regression. Cancer Cell 6: 297-305, 2004.

20. Andrews EJ, Wang JH, Winter DC, Laug WE and Redmond HP. Tumor cell adhesion to endothelial cells is increased by endotoxin via an upregulation of beta-1 integrin expression. J Surg Res 97: 14-19, 2001.

21. Shin SR, Sanchez-Velar N, Sherr DH and Sonenshein GE: 7,12dimethylbenz(a)anthracene treatment of a c-rel mouse mammary tumor cell line induces epithelial to mesenchymal transition via activation of nuclear factor-kappaB. Cancer Res 66: 2570-2575, 2006.

22. Kuphal S, Poser I, Jobin C, Hellerbrand C and Bosserhoff AK: Loss of E-cadherin leads to upregulation of NFkappaB activity in malignant melanoma. Oncogene 23: 8509-8519,2004

23. Vakkila $\mathbf{J}$ and Lotze MT: Inflammation and necrosis promote tumour growth. Nat Rev Immunol 4: 641-648, 2004. 